\title{
OPTICAL CHARACTERIZATION OF THE LASER-INDUCED IGNITION SPARK IN A CRYOGENIC ROCKET COMBUSTOR
}

\author{
Robert G. Stützer, ${ }^{*}$ Michael Börner, \& Michael Oschwald
}

\author{
DLR German Aerospace Center, Lampoldshausen, Baden-Württemberg, 74239, \\ Germany
}

*Address all correspondence to: Robert G. Stützer, DLR German Aerospace Center, Lampoldshausen, 74239, Germany; Tel.: +49 629828 548; Fax: +49 629828 175,

E-mail: robert.stuetzer@dlr.de

Original Manuscript Submitted: XX/X/2017; Final Draft Received: XX/X/2017

\begin{abstract}
Experiments at the European Research and Technology Test Bench P8, located at the DLR Institute of Space Propulsion, were carried out to validate the feasibility of laser ignition on the research rocket combustor "BKA." Propellant injection in BKA was carried out via 15 coaxial injectors with liquid oxygen as oxidizer and gaseous hydrogen or gaseous methane as fuel. Crucial parameters such as the equivalence ratio between oxidizer and fuel (ROF), plasma temperature, ignition delay time, and breakdown threshold can be determined using appropriate methods for plasma analyses. The research rocket combustion chamber "Brennkammer A" (BKA) was equipped with a laser ignition facility and with the opportunity to evaluate the optical emission of the ignition spark. Furthermore, a laboratory-scale cavity, filled with well-defined samples, was used to analyze the plasma lifetime of gaseous samples of known properties. Thus, the local ROF inside the ignition kernel was derived.
\end{abstract}

KEY WORDS: laser ignition, laser-induced breakdown spectroscopy, LIBS, plasma, combustion, space propulsion, rocket combustor, ignition

\section{INTRODUCTION}

Due to the ability to accurately specify the location and time of ignition, laser ignition is considered a versatile method to start combustion in space propulsion. Hence, the application of highenergy lasers becomes increasingly significant. Recently, the German Aerospace Center (DLR Lampoldshausen) made efforts towards the establishment of laser ignition using a research combustor for cryogenic propellants (Börner et al., 2015; Stützer et al., 2015, 2017). The generated ignition spark, induced by a focused laser beam, reveals various physical and chemical properties at the exact place of ignition. Though the overall conditions inside a combustion chamber are well known due to sophisticated simulation tools and numerous experiments, the precise knowledge about the exact time and place of ignition is scarce. Therefore, the optical emission of the ignition spark is used to investigate the ignition process. Crucial parameters such as the ratio of oxidizer and fuel (ROF) can be derived taking advantage of the relative intensities of the atomic emission lines originating from the ion-electron recombinations within the plasma volume. Measurements on gas mixtures with a known stoichiometry are used to obtain calibration lines. Laser-induced breakdown spectroscopy (LIBS) measurements on the ignition spark 
of $\mathrm{LOX} / \mathrm{gH}_{2}$ and $\mathrm{LOX} / \mathrm{gCH}_{4}$ revealed the time from propellant injection until ignition. Furthermore, localized equivalence ratios can be derived and compared to the calibration line. Moreover, a plasma breakdown is accompanied by an abrupt and rapid thermal expansion and heat transfer to the surrounding propellant. According to Boltzmann's law the occupied and free electron states are temperature dependent distributed. Hence, thermodynamic parameters can be deduced from the plasma radiation (Zhang et al., 2014). The factors influencing laser-induced plasma (LIP) temperature include laser pulse characteristics (e.g., laser wavelength, pulse frequency, laser energy, laser mode) (Rusak et al., 1997), ambient surrounding, sample characteristics, and sample geometry. Other parameters such as electron density and the number densities of emitting species can also be determined using Saha-Boltzmann statistics (Stavropoulos et al., 2004).

In order to obtain reliable plasma temperature values, it is important to ensure thermal equilibrium conditions. However, due to the strongly inhomogeneous temperature distribution and short periods of plasma lifetime, only local thermal equilibrium (LTE) conditions can be achieved. The state of the entire system composed of all kinds of species can be described by a series of equilibrium distribution laws (Harilal et al., 2005). For this study, the linearity method was chosen. The closer the data points of the Boltzmann plot are to the linear fit, the more probable the state of LTE is. The Boltzmann distribution law can be applied to describe the relative population of excited levels of an ion (Christoforetti et al., 2010):

$$
N_{n}=N \frac{g_{n}\left(\exp -E_{n} / k_{B} T\right)}{U(T)}
$$

Criteria for appropriate selections of atomic emission lines of the plasma for sufficient temperature measurements and other determinations can be found in the literature (Harilal et al., 2005; Castle et al., 1997). For this study the following requirements had to be fulfilled:

1. The lines must have a reasonable line-to-background ratio.

2. The spectral efficiency should be measured accurately.

3. The greater the gap between the upper energy levels of the selected lines, the better the accuracy of temperature measurement.

4. The accuracy of spontaneous transition probability is relatively high.

5. Special care should be taken to avoid the lines of self-absorption resonance of low-lying energy levels.

Since the spectral output of plasma is dominated by continuous background radiation in the first tens of nanoseconds of the plasma lifetime, the optimal time for spectral measurements has to be found experimentally. A good comparison of methods for LIP temperature measurements can be found in the literature (Hahn and Omenetto, 2010). The Boltzmann-plot method is a straightforward way to determine the plasma temperature. In order to develop and validate temperature measurement software, helium was chosen as the sample gas due to its overwhelming advantages for LIBS experiments. Helium provides a variety of atomic emission lines in the most useful spectral regime that ranges from ultraviolet up to the near-infrared photon wavelengths. The spectrum of helium plasma is characterized by an outstanding signal-to-noise ratio. Hence, it is possible to measure plasma emission for very low pressure values and short exposure times. The chemical inertness makes helium a sample that is safe and easily to handle. 


\section{EXPERIMENTAL}

Several settings have been used for experiments on LIP. On the one hand, data were collected during a laser ignition test campaign using a research rocket combustor and cryogenic propellants, and, on the other hand, laboratory LIBS experiments have been conducted on gas mixtures as well as on solitary gases at ambient temperature conditions and pressures ranging from 0.1 bar up to 10 bar. The latter experiment on ideal conditions was conducted in order to validate software for the rocket combustor test runs and for a better understanding of the processes that occur during the plasma lifetime.

\subsection{Laser Ignition Tests}

Hot firing tests with two different propellants were carried out on the European Research and Technology Test Bench P8 (Haberzettl et al., 2000) at the DLR Institute of Space Propulsion in Lampoldshausen, Germany. Using $\mathrm{gH}_{2}$ or $\mathrm{gCH}_{4}$ as fuel and $\mathrm{LOX}$ as oxidizer, several test runs were conducted. Each test run involved 60 ignition tests with laser bursts, consisting of 20 single laser pulses, fired with a frequency of $50 \mathrm{~Hz}$, a pulse width of 1-2 ns, and a peak energy of $30 \mathrm{~mJ}$. The applied laser ignition system was a Q-switched HiPoLas ${ }^{\circledR}$ laser made by CTR (Carinthian Tech Research AG). The optical path of the laser focused on the shear layer between LOX and gaseous fuel. Propellant injection took place via 15 coaxial injectors that were located on the back of the combustion chamber (see Fig. 1). This segmented, cylindrical combustor was equipped with dynamic pressure sensors throughout its length. The cylindrical element of the combustion chamber closest to the injector plate, however, contained all optical and static pressure sensors as well as the optical laser access (see Fig. 2). One optical probe pointed to the place of plasma breakdown. The optical probes were made of stainless steel and robust sapphire rods, which guaranteed transparency for UV up to NIR wavelengths (Gröning et al., 2015).

In order to monitor the LIP highly time-resolved, photomultipliers were mounted to probe ports pointing into the combustor (see Fig. 2). A breakdown control probe was equipped with an optical band-pass filter for $(306 \pm 5) \mathrm{nm}$ in order to collect the $\mathrm{OH}^{*}$ emission. Further description of the combustion chamber and the test run setup can be found elsewhere (Börner et al., 2015).

LIBS on the ignition spark was conducted using two spectroscopic systems for simultaneous recordings of highly sspectrally resolved data as well as overall pictures ranging from UV to NIR. The plasma signal was split via a fiber switch and both ends were connected to the spectrographs. Using a control voltage signal of the laser diode the pulse generator and therefore both spectrographs were triggered simultaneously. The Acton spectrograph SP2750 of Princeton Instruments ${ }^{\mathrm{TM}}$ was used for the requirement of higher spectral resolution. It had a focal length of $750 \mathrm{~mm}$, a scan range of $0-1400 \mathrm{~nm}$, and was arranged in a Czerny-Turner design, which means
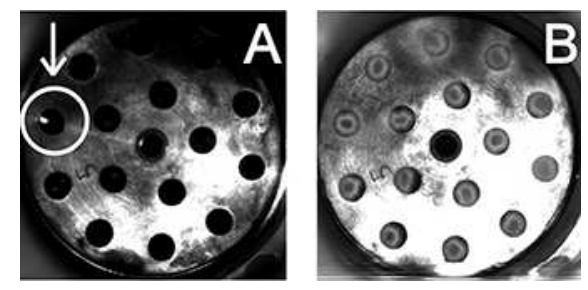

FIG. 1: View into the combustion chamber during laser ignition. A: Plasma cloud on the $\mathrm{LOX} / \mathrm{gH}_{2}$ shear layer (white circle) of one of the 15 coaxial injectors at time $\mathrm{t}_{0}$. B: stable combustion $720 \mathrm{~ms}$ after $\mathrm{t}_{0}$

Volume 17, Issue 1, 2018 


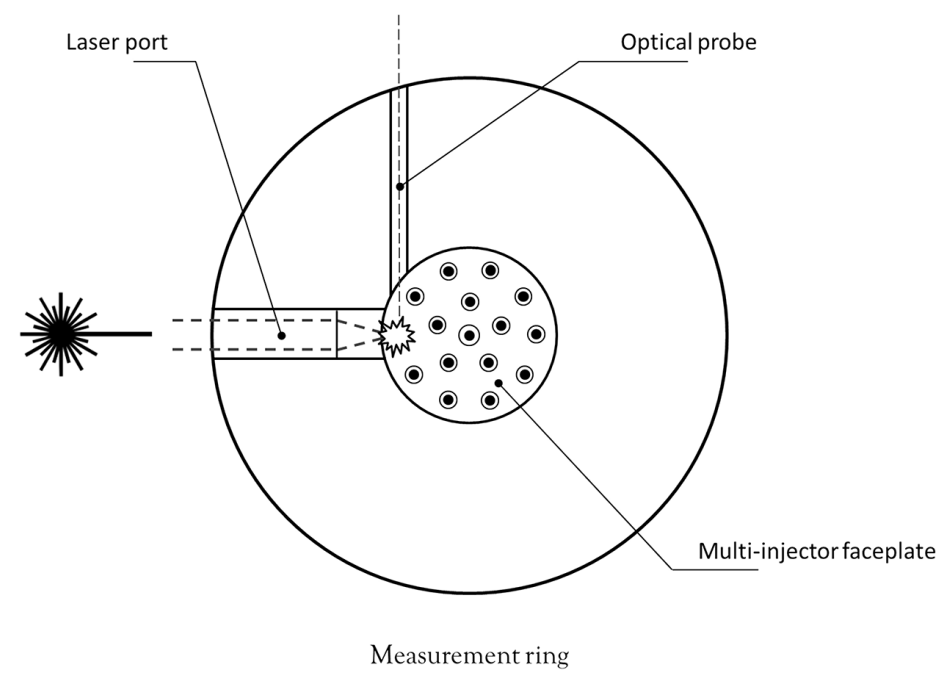

FIG. 2: Diagnostic segment (front view) as applied in combustion chamber A (BKA) for optical and pressure measurements. The laser-induced ignition spark was located at an outer coaxial injector with the possibility for optical plasma investigation (LIBS).

the refractive element is a linear grating that is placed between two concave mirrors. Optical signals were recorded via the intensified camera PI-MAX: $1024\left(\mathrm{PI}^{\mathrm{TM}}\right)$ with a front illuminated, Peltier-cooled, $1012 \times 256$ imaging array (pixel size: $26 \mu \mathrm{m} \times 26 \mu \mathrm{m}$ ). All measurements were conducted in gated mode for short exposure times and to avoid outshining by the laser intensity. All spectra were recorded using a grating with $150 \mathrm{gr} / \mathrm{mm}$ and a center wavelength of $720 \mathrm{~nm}$ in order to depict emission of the $\mathrm{H} \alpha$ line at $656.3 \mathrm{~nm}$ and of oxygen at $777.2 \mathrm{~nm}$ in a single frame.

Wider range LIBS measurements were carried out using the Czerny-Turner designed Andor ${ }^{\mathrm{TM}}$ Shamrock SR-163 spectrograph with a focal length of $163 \mathrm{~mm}$. A diffraction grating, blazed for $350 \mathrm{~nm}$ and $150 \mathrm{gr} / \mathrm{mm}$, was applied in order to obtain the entire spectrum from a 250- to 900-nm wavelength in a single exposure.

A Dalsa ${ }^{\circledR}$ Genie-HM 1024 surveillance camera was mounted in front of the nozzle with an unobstructed view to the place of ignition. The camera possessed a monochromatic $2 / 3$ " CMOS sensor with a global shutter and $1024 \times 768$ pixels. The sequence of interest was recorded with the maximum frame rate of $117 \mathrm{fps}$. A LED light source was installed behind the camera to illuminate the combustion chamber faceplate. Both camera and light source were encased by a steel housing with a 2-cm-thick quartz glass window on its front end. Convective cooling with gaseous nitrogen prevented the system from overheating when exposed to hot gases of the exhaust plume.

\subsection{Calibration Measurements}

Measurements on well-defined gases and gas mixtures were carried out. A small stainless-steel chamber with a cavity of the size of ca. $10 \mathrm{~cm}^{3}$ was used to retain the gas under investigation. The setup is depicted in Fig. 3. The ratio of the components was controlled via pressure sensors in order to adjust correct partial pressure values. Calibration experiments for the laser ignition campaign were conducted using $\mathrm{gH}_{2}$ and $\mathrm{gO}_{2}$. The total, absolute pressure was set either at 


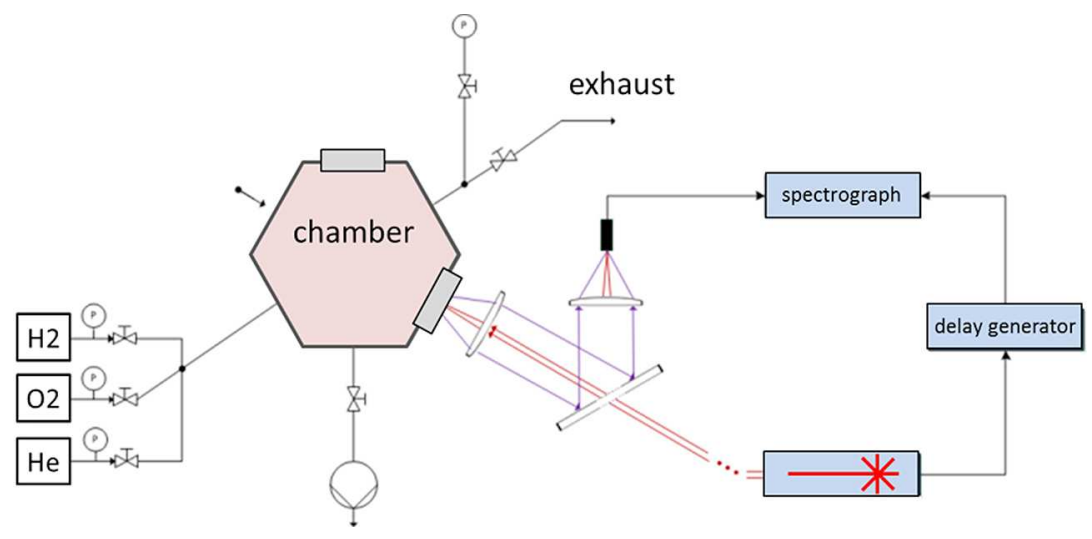

FIG. 3: LIBS setup for nonablative plasma spectroscopy

500 mbar or 1 bar. Furthermore, binary mixtures of the four gases hydrogen, oxygen, helium, and argon were used in order to measure calibration lines regarding spectral output as a function of mixture ratio.

In order to develop and evaluate software for plasma temperature and breakdown threshold measurements, helium was used due to its outstanding spectral properties (see Fig. 4). Helium is nontoxic and nonreactive by its characteristic nature which allows safe plasma experiments without any hazards. Helium quantum state configuration allowed more than two distinct characteristic emission lines (peaks) in its spectrum. This allowed the application of the Boltzmann-plot method for plasma temperature determination. Helium offered an excellent signal-to-noise ratio regarding LIBS.

For this experiment a water-cooled Nd:YAG Quantel ${ }^{\mathrm{TM}}$ laser system was applied in order to induce the ignition plasma in the gas chamber (nonablative) using its fundamental wavelength of $1064 \mathrm{~nm}$ and pulse energies between 30 and $300 \mathrm{~mJ}$. Due to its ignitability, only one laser-induced breakdown event was used for a LIBS measurement in the respective hydrogen/oxygen gas composition. For experiments on inert gas mixtures, between 50 and 100 plasma emissions were used to analyze one measurement point (mixture ratio). The primary beam diameter was less than $10 \mathrm{~mm}$. The dichroic mirror enabled coaxial access into the cavity and quartz glass lenses focused the laser beam on the location of breakdown. The Gaussian line shape of the applied laser beam was evaluated using time-resolved calorimetric measurements.

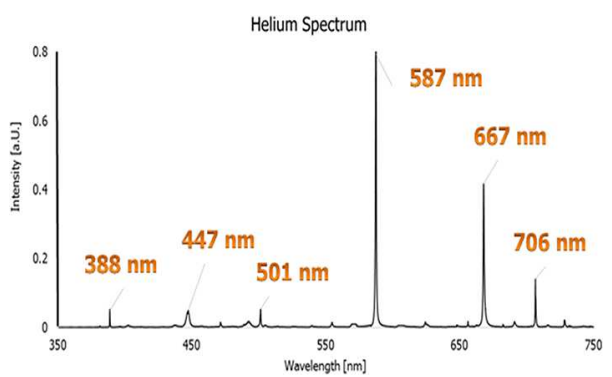

FIG. 4: Spectrum of a typical helium plasma emission with its prominent transition lines

Volume 17, Issue 1, 2018 
The chamber, designed for pressures up to 15 bar, had several gas connections and was optically accessible using quartz glass windows. The laser beam was focused into the cavity by a lens with $f=150 \mathrm{~mm}$ in order to create a volume for breakdown of $1 \mathrm{~mm}^{3}$ in the gas phase (nonablative). A dichroic mirror (Thorlabs ${ }^{\circledR}$ ) with a cutoff wavelength of $950 \mathrm{~nm}$, filtering the laser wavelength of $1064 \mathrm{~nm}$, allowed coaxial application of the laser beam and the plasma emission. The latter was focused on the optical fiber by a $f=40 \mathrm{~mm}$ plano-concave lens. Using the SP2750 spectrograph of Princeton Instruments ${ }^{\mathrm{TM}}$, the plasma emission was spectrally analyzed.

\section{RESULTS}

\subsection{Laser Ignition}

As depicted in Fig. 1, the focused laser induced plasma on the boundary layer between LOX and gaseous fuel in order to ignite all 15 injectors. A typical response of the optical and pressure data is shown in Fig. 5. Clearly visible is the laser plasma emission during the first $500 \mathrm{~ms}$ of a $\mathrm{LOX} / \mathrm{gH}_{2}$ ignition. The subsequent, rather noisy signal is the emission of $\mathrm{OH}^{*}$ radicals and therefore a direct indicator for the combustion process. The corresponding pressure data are shown in the red graph, revealing a distinctive match between both optical and pressure data sets. This is in excellent agreement with results of former investigations on hydrogen flames (Fiala et al., 2017). Moreover, fast Fourier transform (FFT) analysis also showed identical behavior for optical and pressure responses. Hydrogen flames exhibit a dominant frequency at $950 \mathrm{~Hz}$. On the other hand, methane flames show four distinct frequencies at 950, 1750, 3600, and 5450 $\mathrm{Hz}$ for this experimental setup (Stützer et al., 2015). Figure 6 shows optical emission spectra of the electron-ion recombination after plasma breakdown. These LIBS measurements show only hydrogen emission for the first five laser pulses. As seen in Fig. 5, no combustion takes place during the first $500 \mathrm{~ms}$. The most prominent hydrogen line is the $\mathrm{H} \alpha$ line at $656.3 \mathrm{~nm}$. Spectral measurements using broader ranges also showed $\mathrm{H} \beta$ as well as $\mathrm{H} \gamma$ lines which have been used for temperature measurements (Stützer et al., 2016). However, the ratio between $\mathrm{H} \alpha$ and the O I triplet is nearly constant over a wide range of laser pulse energy values, which is in accordance with the literature (Phuoc and White, 2002).

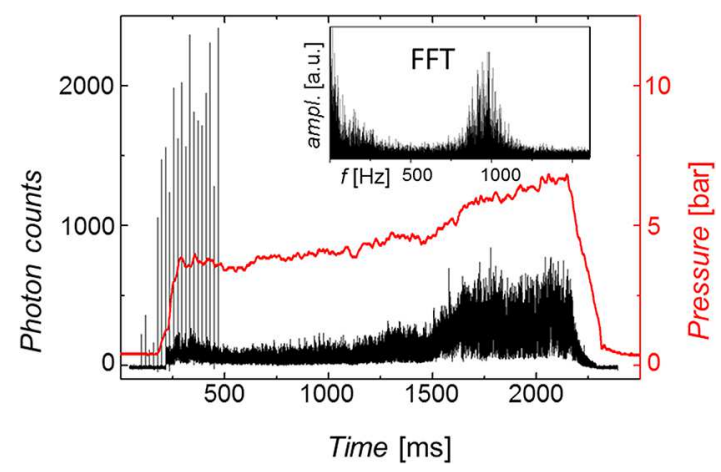

FIG. 5: Typical sequence of a laser-ignited $\mathrm{LOX} / \mathrm{gH}_{2}$ combustion. The black line depicts the optical signal with the laser-induced plasma emission peaks during the first $500 \mathrm{~ms}$. The red line is the corresponding chamber pressure. Inlay: both optical and pressure data show a dominant frequency at $950 \mathrm{~Hz}$. 


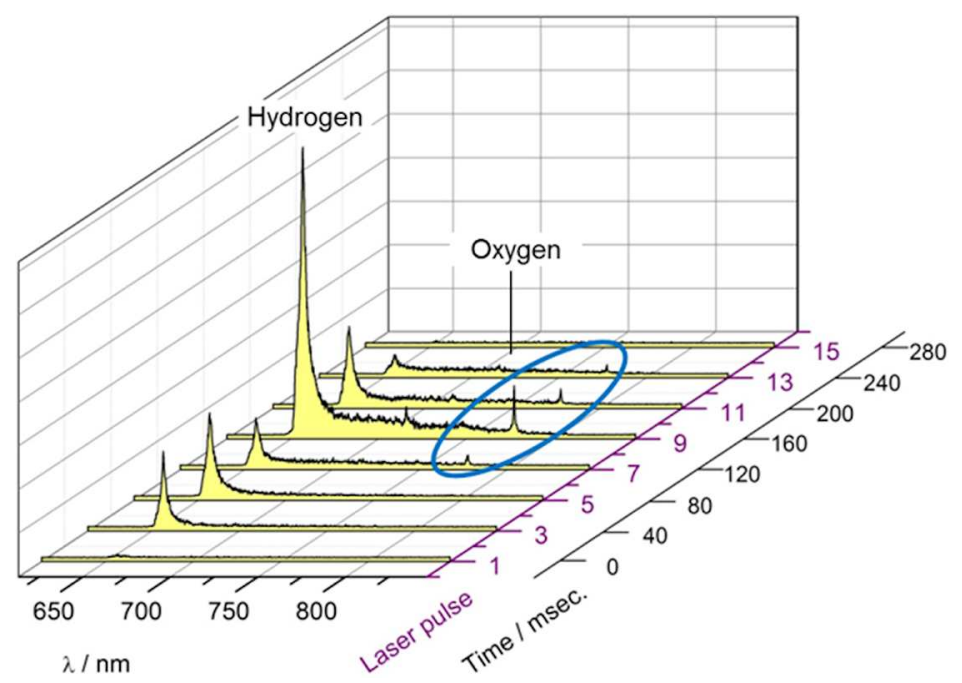

FIG. 6: LIBS measurements on ignition sparks during $\mathrm{LOX} / \mathrm{gH}_{2}$ injection

\subsection{Plasma Temperature Determination}

In order to determine reliable plasma temperature values it is indispensable to ensure local thermal equilibrium (LTE) conditions within the measured volume and time. Hence, the number of electrons excited to higher levels and their falling back to lower levels are balanced. The Boltzmann method is a convenient means of determining the excitation temperatures in laser-induced plasma when LTE is established. Using the intensities and wavelengths corresponding to the specific transitions in a spectrographic measurement of the plasma, and applying appropriate degeneracies and Einstein coefficients corresponding to the probability of spontaneous emission, the data points for Boltzmann and Saha-Boltzmann plots can be calculated. The slope of a linear regression fit for these data points gives the excitation temperature. For a transition from the higher energy level $E_{n}$ to the lower energy level $E_{m}$, the intensity of the transition can be expressed by the following equation:

$$
I_{n m}=\frac{h c N_{o} g_{n} A_{n m}}{Q \lambda_{n m}} \exp \left(\frac{-E_{n}}{k T}\right)
$$

Here, $h$ is the Planck constant, $c$ is the velocity of light, $N_{o}$ is the number of free atoms, $g_{n}$ is the statistical weight of the level $E_{n}, Q$ is the partition function of the atom, $A_{n m}$ is the transition probability for spontaneous emission, $\lambda_{n m}$ is the wavelength of the spectral line, $k$ is the Boltzmann constant, and $T$ is the absolute temperature. A good measure to estimate the quality of the LTE is the deviation of the data points from the average Boltzmann line (linearity). The more linearly the data points are arranged in the Boltzmann plot the more reliable are the LTE conditions. As seen in Fig. 7, LTE quality strongly depends on the time between the laser pulse itself and the measurement. Best LTE conditions can be obtained for a delay time between 2 and $3 \mu$ s.

Further experiments on helium plasma were conducted in order to evaluate the influence of pressure as well as laser pulse energy on the plasma temperature using the Boltzmann-plot method. Figure 8 shows the laser-induced helium plasma values as a function of pressure for

Volume 17, Issue 1, 2018 


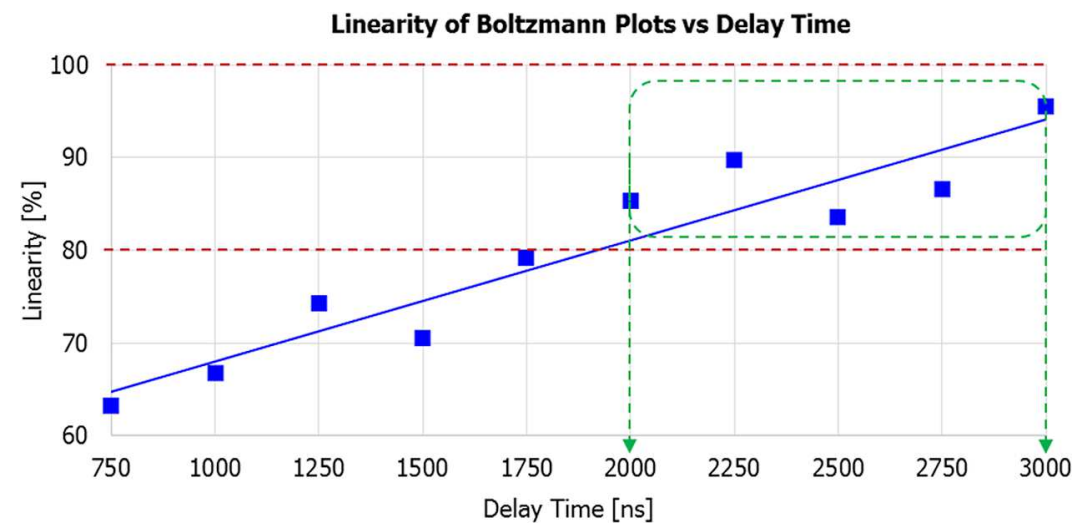

FIG. 7: Linearity in the Boltzmann plots versus delay time (time between laser pulse and measurement)

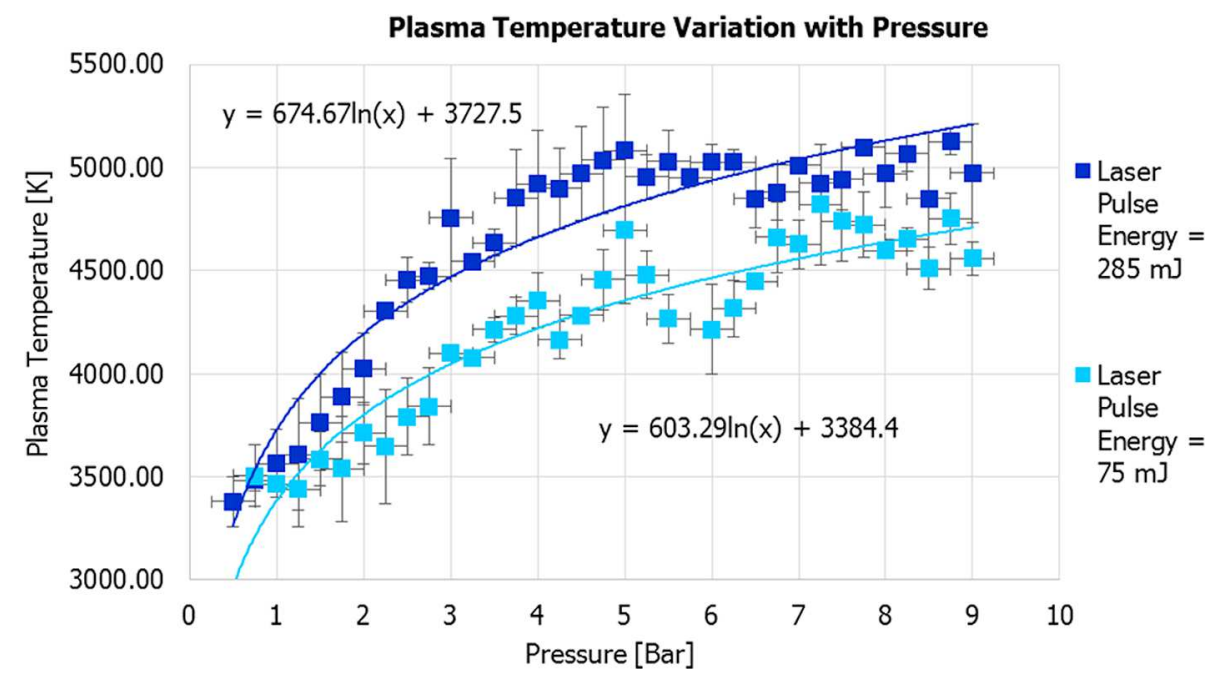

FIG. 8: Helium plasma temperature as a function of pressure and laser pulse energy

two different laser pulse energy levels. Obviously higher laser pulse energy results in higher plasma temperature. The highest plasma temperatures of $5100 \mathrm{~K}$ were reached for more than $5 \mathrm{bar}$ and a laser pulse energy of $285 \mathrm{~mJ}$. Furthermore, the plasma temperature function seems to be invariant for higher pressure values. All parameters have been set for best signal-to-noise ratios.

In order to obtain critical laser pulse energy levels for plasma breakdown creation, the number of plasma events was counted for a series of laser pulse energy and two different pressure values. Results are shown in Fig. 9. Regarding the relative number of plasma breakdown events, there are three different laser pulse energy regimes: The regime with the lowest laser pulse energy is characterized by a lack of plasma events, the intermediate energy regime shows a certain percentage of laser pulses that are able to create a breakdown in helium, and within the highest energy regime all laser pulses create a plasma breakdown in the helium gas. Moreover, increasing pressure clearly results in a shift of the regimes towards lower laser pulse energy values. 


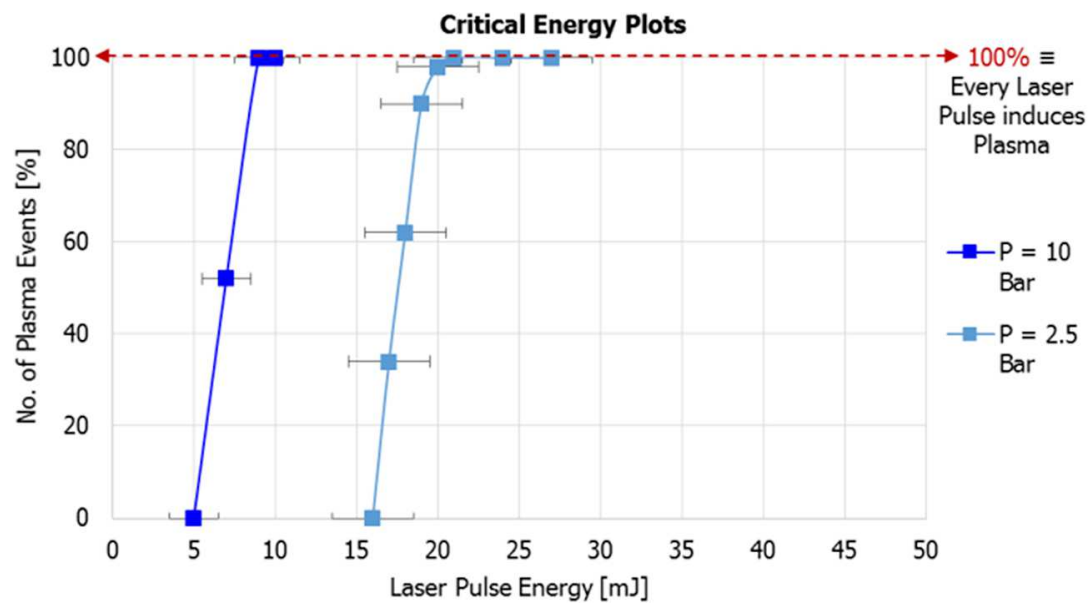

FIG. 9: Critical laser pulse energy. Number of plasma breakdown events as a function of laser pulse energy for $\mathrm{P}=10$ bar and $\mathrm{P}=2.5$ bar

All three breakdown regimes are depicted in Fig. 10 in more detail as a function of pressure. The actual critical laser pulse energy value lies in the middle between the green and the red data points.

Due to the ideal spectral properties of helium, the results of LIBS experiments on this noble gas are suitable for calibration measurements and software validation. Thus, using the helium references for hydrogen and oxygen, plasma temperature values were obtained from measurements of plasma emission during laser ignition of gaseous propellant combustion. The Balmer series of hydrogen is an excellent candidate for plasma temperature measurements due to its intense emission lines in the visible range of the spectrum. These atomic transition lines thus are

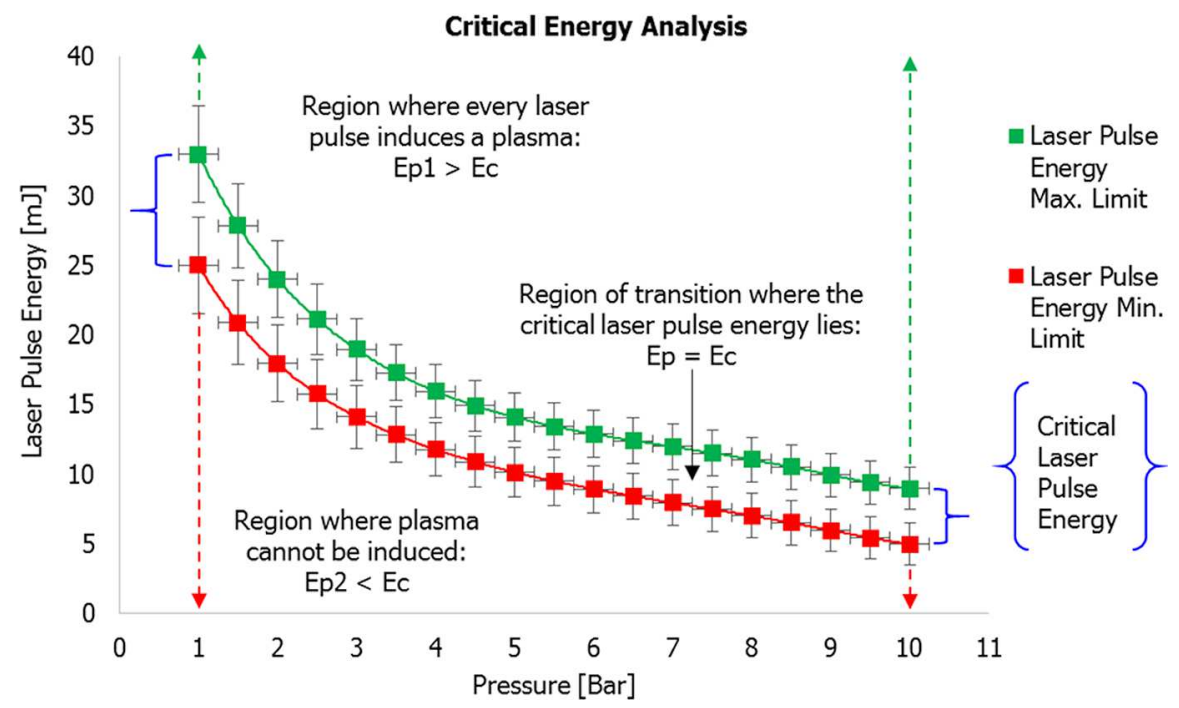

FIG. 10: Critical laser pulse energy versus pressure

Volume 17, Issue 1, 2018 
suitable for the Boltzmann-plot method. Figure 11 depicts an optical spectrum of hydrogen with the three brightest lines of the Balmer series ( $\mathrm{H} \alpha: 656.3 \mathrm{~nm} ; \mathrm{H} \beta: 486.1 \mathrm{~nm} ; \mathrm{H} \gamma: 434.0 \mathrm{~nm}$ ), which are clearly seen during the laser ignition experiments.

Hence, the slope of the average line of the three atomic lines in the Boltzmann plot delivers the plasma temperature. Using the relation

$$
T=\frac{1}{|m| k_{B}}
$$

with slope $=m$, the temperature of the plasma is the reciprocal coefficient with the Boltzmann constant. The decline in Fig. 12 gives the absolute value of $(1.3 \pm 0.02) \mathrm{eV}$ and thus a plasma temperature of $(8939 \pm 137) \mathrm{K}$.

\section{CONCLUSIONS}

The feasibility of laser ignition of a LOX/gH2 injection was demonstrated. The goal, the evaluation of plasma temperature data and the measurement of the ratio of oxidizer and fuel (ROF) at the exact place of ignition, was reached using laser-induced breakdown spectroscopy (LIBS) on the ignition sparks. For software development and validation LIBS experiments were carried out on helium under several pressure values and on well-defined hydrogen-oxygen gas samples with different mixture ratios. The focus of the helium experiments was finding appropriated delay

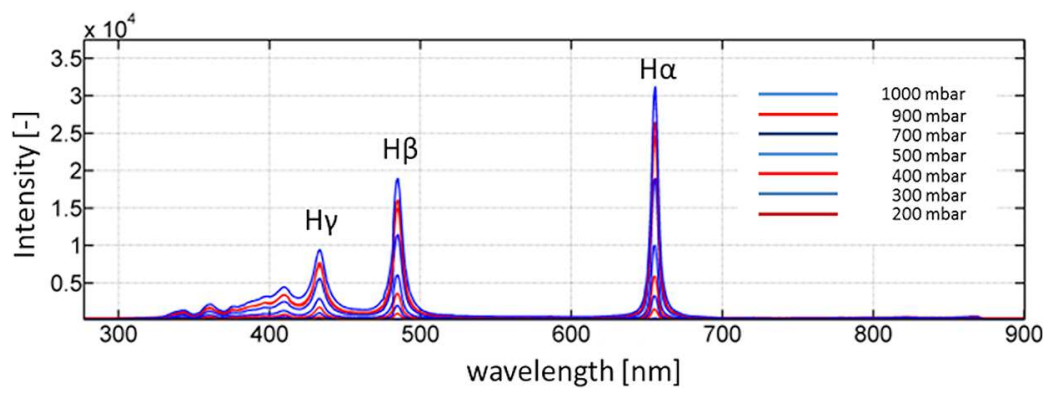

FIG. 11: Hydrogen plasma emission for several pressure values. The three most distinct atomic emission lines of the Balmer series $(\mathrm{H} \alpha, \mathrm{H} \beta, \mathrm{H} \gamma)$ can be used for plasma temperature determination.

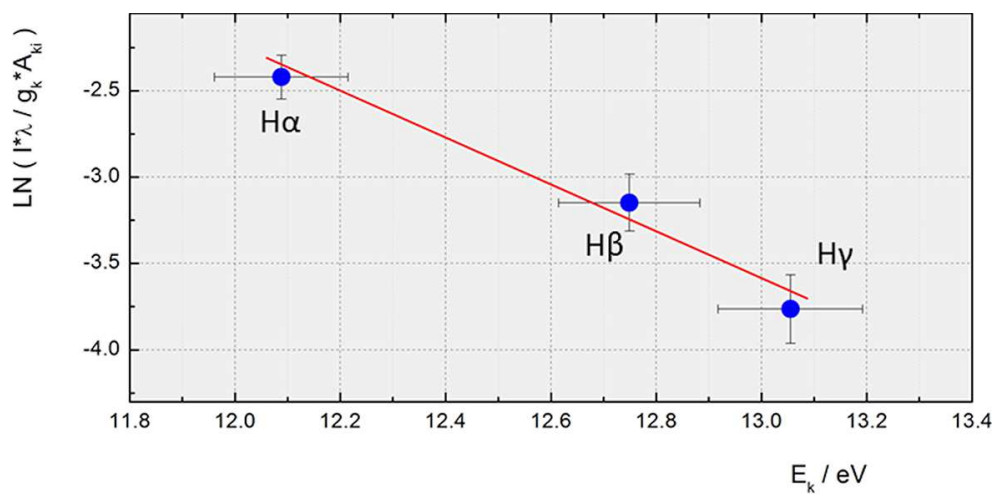

FIG. 12: Boltzmann plot of hydrogen emission lines (Balmer series) for plasma temperature determination 
and exposure times for optimal local thermal equilibrium (LTE) conditions during the plasma lifetime. The influence of pressure and laser pulse energy $\left(E_{\text {pulse }}\right)$ on the measurement parameters was also of interest and it was found that both pressure and $E_{\text {pulse }}$ had neither influence on the plasma life time nor on the time for optimal LTE conditions. On the other hand, the plasma temperature increased with both higher pressure values and higher $E_{\text {pulse }}$.

\section{REFERENCES}

Börner, M., Manfletti, C., Grebe, A., Hardi, J., Stützer, R., Suslov, D., and Oschwald, M., (2015) Experimental Study of a Laser-ignited Liquid Cryogenic Rocket Engine, in LIC-15-Th2A.5, Proc. of 4th Laser Ignition Conf. (LIC), Argonne, IL.

Castle, B., Visser, K., Smith, B., and Winefordner, J., (1997) Level Population in a Laser-induced Plasma on a Lead Target, Spectrochim. Acta, Part B, vol. 52, pp. 1995-2009.

Christoforetti, G., Lorenzetti, G., Legnaioli, S., and Palleschi, V., (2010) Investigation on the Role of Air in the Dynamical Evolution and Thermodynamic State of a Laser-induced Aluminium Plasma by Spatialand Time-Resolved Spectroscopy, Spectrochim. Acta, Part B, 65, pp. 787-796.

Fiala, T., Sattelmayer, S., Gröning, S., Hardi, J., Stützer, R., Webster, S., and Oschwald, M., (2017) Comparison between $\mathrm{OH}^{*}$ and Blue Radiation from Hydrogen Rocket Combustion, J. Propuls. Power, 33, pp. 490-500.

Gröning, S., Hardi, J., Suslov, D., and Oschwald, M., (2015) Analysis of Phase Shift between Oscillations of Pressure and Flame Radiation Intensity of Combustion Instabilities, in Proc. of 6th European Conf. for Aeronautics and Space Sciences (EUCASS), Krakow, Poland.

Haberzettl, A., Gundel, D., Bahlmann, K., Thomas, J., Kretschmer, J., and Vuillermoz, P., (2000) European Research and Technology Test Bench P8 for High Pressure and Liquid Rocket Propellants, in Proc. of 36th Joint Propulsion Conf. and Exhibit (JPC), Huntsville, AL, pp. 1-13.

Hahn, D. and Omenetto, N., (2010) Laser-induced Breakdown Spectroscopy (LIBS), Part I: Review of Basic Diagnostics and Plasma-Particle Interactions, Appl. Spectrosc., 64(12), pp 335-366.

Harilal, S., O’Shay, M., Tillack, M., and Mathew, M., (2005) Spectroscopic Characterization of LaserInduced Tin Plasma, J. Appl. Phys., 98, p. 013306.

Phuoc, T. and White, F., (2002) Laser-induced Spark for Measurements of the Fuel-to-Air Ratio of a Combustive Mixture, Fuel, 81, pp. 1761-1765.

Rusak, D.A., Castle, B.C., Smith, B.W., and Winefordner, J.D., (1997) Excitational, Vibrational, and Rotational Temperatures in Nd: YAG and XECL Laser-induced Plasmas, Spectrochim. Acta, Part B, 52, pp. 1929-1935.

Stavropoulos, P., Palagas, C., Angelopoulos, G., Papamantellos, D., and Couris, S., (2004)Calibration Measurements in Laser-induced Breakdown Spectroscopy Using Nanosecond and Picosecond Lasers, Spectrochim. Acta, Part B, 59, pp. 1885-1892.

Stützer, R., Börner, M., and Oschwald, M., (2015) Investigation of a Laser-ignited Cryogenic Rocket Cumbustion, in Proc. of 6th European Conf. for Aeronautics and Space Sciences (EUCASS), Krakow, Poland.

Stützer, R., Börner, M., Kuchelmeister, M., and Oschwald, M., (2016) Laser-induced Breakdown Spectroscopy on Gas Compositions for Equivalence Ratio Determination in Space Propulsion, in Proc. of 5th Space Propulsion Conf., Rome, Italy.

Stützer, R., Börner, M., and Oschwald, M., (2017) Laser-induced Breakdown Spectroscopy on the Ignition Spark of a Cryogenic Rocket Combustion, in LIC-17-LFA4.2, Proc. of 5th Laser Ignition Conf. (LIC), Bucharest, Romania.

Zhang, S., Wang, X., He, M., Jiang, Y., Zhang, B., Hang, W., and Huang, B., (2014) Laser-induced Plasma Temperature, Spectrochim. Acta, Part B, 97, pp. 13-33.

Volume 17, Issue 1, 2018 